\title{
Hybrid modelling case studies
}

\author{
Rosemarie Sadsad, ${ }^{1}$ Geoff McDonnell, ${ }^{2}$ Joe Viana, ${ }^{3}$ \\ Shivam M. Desai, ${ }^{4}$ Paul Harper ${ }^{5}$ and Sally Brailsford ${ }^{6}$ \\ ${ }^{1}$ Faculty of Medicine, University of New South Wales, Sydney, Australia \\ ${ }^{2}$ Director UNSW, Bronte, Australia \\ ${ }^{3}$ School of Management, University of Southampton, UK \\ ${ }^{4}$ Department of Management, University of Southampton, UK \\ ${ }^{5}$ School of Mathematics, University of Cardiff, UK \\ ${ }^{6}$ Southampton Management School, University of Southampton, UK
}

\subsection{Introduction}

The objective of this chapter is to demonstrate the use of hybrid modelling for management decision support. The chapter presents three separate case studies that are unified both by the common theme of using different modelling techniques in a hybrid manner and by the health and social care context.

The first case study combines the use of system dynamics and agent-based modelling to better understand and control the spread of a specific drug-resistant pathogen in hospitals; the second case study demonstrates how a hybrid system dynamics and discrete-event simulation model can support clinical and management decision making in the context of sexual health services; and the third case study is dedicated to the investigation of a hybrid model that consists of a system-dynamics-inspired cell-based population model and a discrete-event simulation model, and is used to explore the performance of the contact centre for long-term care for people aged 65 and over.

Discrete-Event Simulation and System Dynamics for Management Decision Making, First Edition. Edited by Sally Brailsford, Leonid Churilov and Brian Dangerfield.

(C) 2014 John Wiley \& Sons, Ltd. Published 2014 by John Wiley \& Sons, Ltd. 


\subsection{A multilevel model of MRSA endemicity and its control in hospitals}

\subsubsection{Introduction}

A health-care-associated infection is an infection acquired by patients while receiving medical treatment in a health care facility (CDC, 2011; WHO, 2011). Health-careassociated infections are the most common complication to affect patients in hospitals; most cases are preventable (WHO, 2011). Many infections are caused by endemic and often drug-resistant pathogens such as methicillin-resistant Staphylococcus aureus (MRSA) (Gould, 2005; Grundmann et al., 2006; Köck et al., 2010). MRSA acquired during hospital stay is associated with significant morbidity and mortality and places a large burden on health care resources. Worldwide, hospital infection control has had mixed success for reducing hospital-acquired MRSA infections, highlighting the challenge of identifying the most effective infection control policy for a given setting and patient mix. In this chapter a conceptual model is developed that provides a multilevel view of MRSA endemicity in hospitals. The model illustrates how factors from different levels of the health system affect MRSA endemicity in hospitals. Key stakeholders and responsibilities towards MRSA management and the areas they may target are identified. The process for how a multiscale simulation model is developed from the conceptual model is described.

\subsubsection{Method}

The conceptual model was developed using several multilevel system theories and frameworks. The merits of the theories and frameworks used are described in detail in Chapter 13. Several determinants of MRSA endemicity in hospitals were described using the context-mechanism-outcome (CMO) framework of the realist approach (Pawson and Tilley, 1997). Two public health outcomes relating to MRSA endemicity in hospitals were of interest. These outcomes are the prevalence of MRSA and the incidence rate of MRSA in a hospital, and are monitored at the health care facility and regional level. The prevalence of MRSA is the total number of people colonised or infected with MRSA at one point in time. The incidence rate of MRSA is the total number of people newly colonised or infected with MRSA over a period of time. The determinants of MRSA endemicity in hospitals were categorised as either mechanisms for the prevalence and/or incidence of MRSA, or contextual factors that could affect the operation of these mechanisms. The levels of the health system, within which the determinants or their relationships exist, were identified to indicate the levels of management and responsibilities involved. These levels were presented as a Stommel diagram (Stommel, 1963) to form a Health System Framework (Ferlie and Shortell, 2001; Glass and McAtee, 2006). The relationships between determinants that existed at the individual level, either within the individual or between individuals, were described with Unified Modeling Language (UML) statecharts (OMG, 2012). The relationships between determinants that exist at the health care facility and community level of the health care system were described using stock and flow 
diagrams of the system dynamics approach (Forrester, 1961; Sterman, 2000). The UML statecharts and stock and flow diagrams were embedded within the Health System Framework, forming a multilevel conceptual model. As discussed in Chapter 13, flows in the stock and flow diagrams and the occurrence of events in UML diagrams are points of leverage health care management can target to change outcomes. Contextual factors may also be targeted or introduced by health care management to control the flow and event mechanisms.

\subsubsection{Results}

Our conceptual model (see Figure 14.1) is based on classic models of infectious disease (Hamer, 1906; Kermack and McKendrick, 1927). In our model, the mechanisms for the prevalence of MRSA are the net admission (patient admissions less discharges and deaths) of patients already colonised or infected with MRSA and the incidence of MRSA. The incidence is conceptualised as both an outcome and mechanism. When the prevalence of MRSA is the outcome of interest, the incidence of MRSA is a mechanism; however, it is also a separate outcome of interest with its own mechanisms and context. Variables may be perceived as a contextual factor, mechanism or outcome based on the perspective from the outcome of interest. The numbers of people susceptible and infectious (colonised or infected) with MRSA are represented as stocks. People entering and leaving the hospital, and new MRSA cases, are represented as flows into, out of and between these stocks, respectively. An individual in the hospital is described with a UML statechart. An individual is in one of two states: susceptible to acquiring MRSA or is infectious, being colonised or infected with MRSA. An individual transitions from the susceptible state to being infectious in the event of successful transmission of MRSA upon contact with another individual colonised or infected with MRSA or their environment. This is the mechanism for a new case of MRSA acquired in hospital in our model. All other variables interact and, together, can control the flow rates and consequently the number of people in the stocks. They can also control the occurrence of MRSA transmission events.

Several contextual factors are shown that affect the mechanisms for the prevalence and incidence of MRSA in hospitals. The contextual factors also reflect the multilevel nature of the problem. At the community level, the prevalence of MRSA among those living in the community may affect the prevalence of MRSA among those admitted to the hospital. At the facility level, bed capacity can limit the number of people admitted to the hospital. At the individual level, high pathogen transmissibility or an individual's infectivity or susceptibility can increase the chance of MRSA transmission upon contact.

The effect of several hospital infection control policies was explored with our model. Our model illustrates that hand hygiene, where MRSA pathogens are removed or reduced from the contaminated hands of people using antimicrobial hand wash, reduces the likelihood of MRSA transmission. All other policies investigated are shown to reduce the number of contacts made between susceptible and infectious people. These policies include: cohorting, where either staff are assigned to particular patients, or infectious patients are allocated to a designated area or isolated in a single-bed room; and active surveillance for MRSA, for example routine screening of patients for MRSA. 


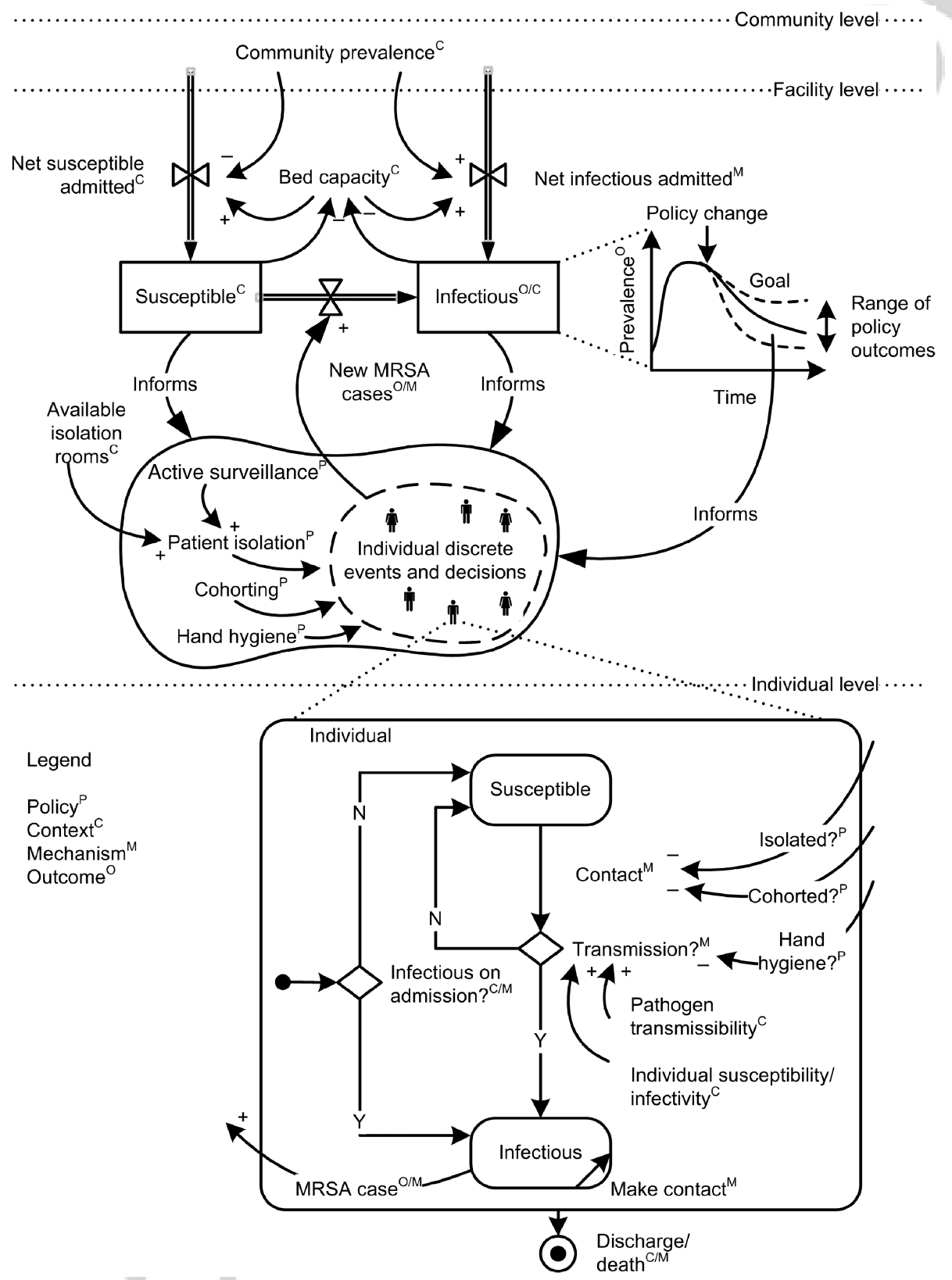

Figure 14.1 A multilevel conceptual model of MRSA endemicity and its control in hospitals. The model was developed by applying the CMO framework of the realist approach (Pawson and Tilley, 1997), stock and flow concepts of the system dynamics approach (Sterman, 2000) and UML statecharts (OMG, 2012). The levels of the health system involved in its management are shown. 
Single-bed room capacity is also shown to limit the number of patients that can be isolated. Our conceptual model portrays the response from individuals to hospital infection control policies. The policies target both the mechanisms and context for the incidence of MRSA, but do not address the prevalence of MRSA directly. Our model suggests community-level strategies that reduce the prevalence of MRSA in the community, as well as facility-level strategies that could reduce the prevalence of MRSA in the hospital and, indirectly, further reduce the incidence of MRSA in the hospital.

The conceptual model provides insight into the determinants of MRSA endemicity in hospitals and the relationships between these determinants. To quantify the magnitude of their impact and describe how these determinants and their relationships change over time, the conceptual model can be translated into a multiscale simulation model. This multiscale simulation model provides a framework to explore alternative scenarios and evaluate infection control policies that may not be feasible to conduct in the real world.

We followed the six-step process for developing multiscale simulation models described in Chapter 13. The conceptual model (see Figure 14.1) shows the health care facility and individuals as being largely responsible for MRSA endemicity in hospitals and its management. Also, hospital-wide infection control policies differ in their implementation across ward specialties. As such, phenomena were modelled at the individual, ward and hospital level.

Three single-scale models were developed individually and then combined using a hierarchical and parallel approach to form the multiscale model (see Figure 14.2):

1. The individual-based model simulates contact events between patients and health care workers and the chance of MRSA transmission based on individual attributes and the infection control precautions taken. This is an agent-based model.

2. The ward model simulates ward-specific settings, infection control policies, patient flow through the ward and patient bed allocation. This is a multimethod

Health System Levels

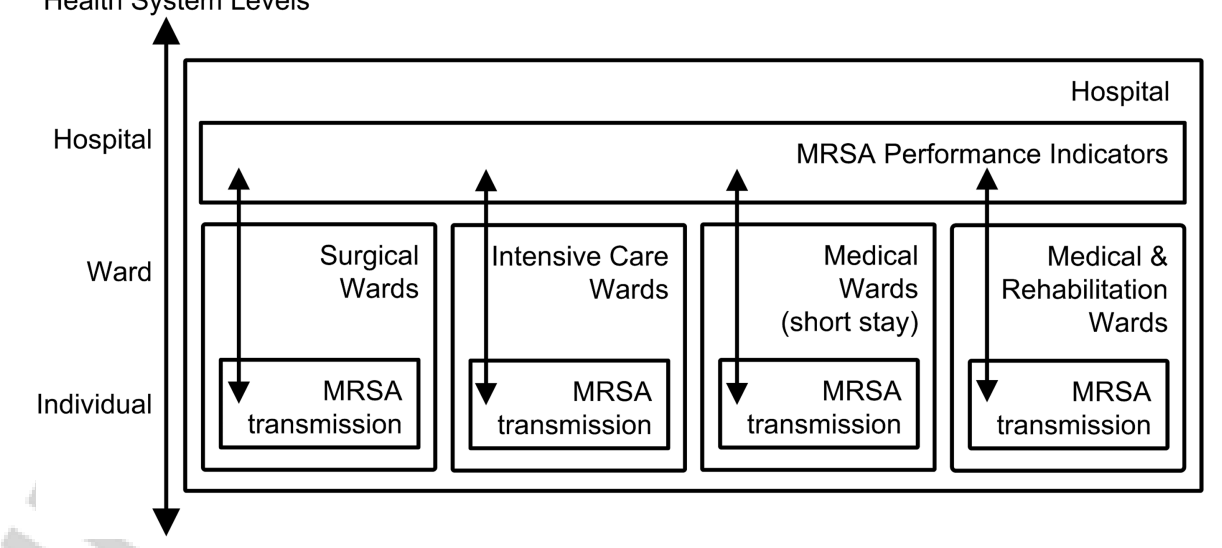

Figure 14.2 The structure of a multiscale model of MRSA endemicity and its control in hospitals. 
(a) Hospital management view

\section{haisim}

Summary

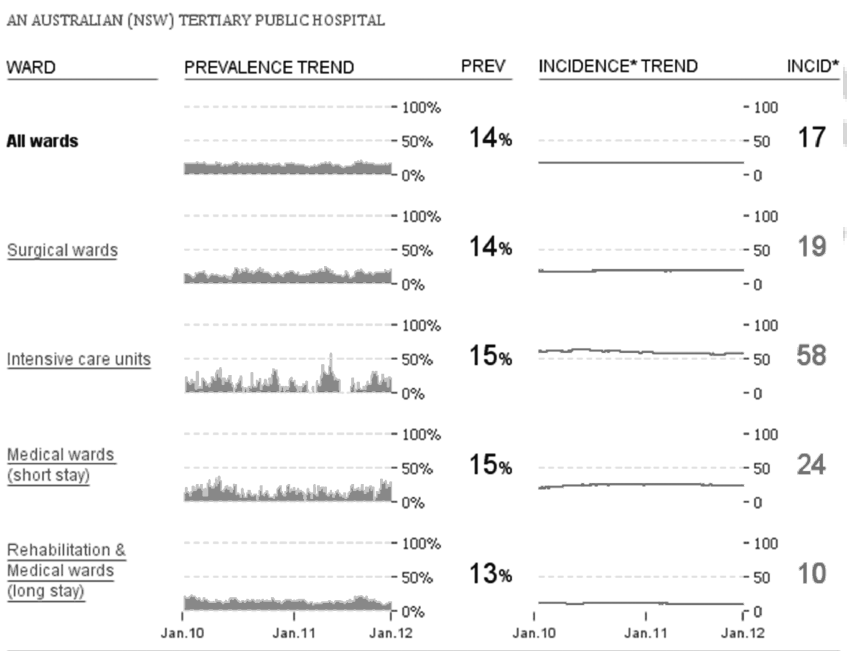

* MRSA incidence rate per 10,000 overnight bed days

(b) Ward management view

\section{haisim}

Intensive care units

$$
\text { SUMMARY STATISTICS }
$$

AVERAGE DAILY AVERAGE DAILY INCIDENCE PREVALENCE (\%) PREVALENCE (N) RATE *

\begin{tabular}{llll}
\hline 15 & 4 & $(\mathrm{SD}=3) \quad 58$
\end{tabular}

MRSA PREVALENCE TREND

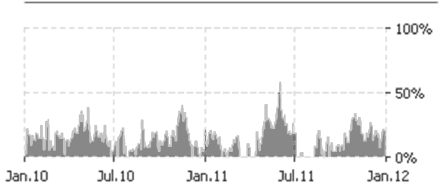

MRSA INCIDENCE RATE* TREND

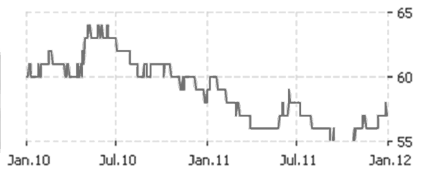

* MRSA incidence rate per 10,000 overnight bed days view all wards

view or edit ward settings

\begin{tabular}{|c|c|c|}
\hline $\begin{array}{l}\text { DAILY } \\
\text { PREVALENCE (\%) }\end{array}$ & $\begin{array}{l}\text { DAILY } \\
\text { PREVALENCE }\end{array}$ & \multirow{2}{*}{$\begin{array}{l}\text { DAILY } \\
\text { INCIDENCE (N) } \\
1\end{array}$} \\
\hline 24 & 5 & \\
\hline \multicolumn{3}{|l|}{ PATIENT LOCATION } \\
\hline & \multicolumn{2}{|c|}{$\begin{array}{l}\text { Shared rooms } \\
\mathbf{0} \text { colonised } \\
\mathbf{0} \text { nosocomial } \\
\mathbf{2} \text { uncolonised } \\
\mathbf{4} \text { empty beds }\end{array}$} \\
\hline & \multicolumn{2}{|c|}{$\begin{array}{l}\text { Single bed rooms } \\
5 \text { colonised } \\
1 \text { nosocomial } \\
14 \text { uncolonised } \\
6 \text { empty beds }\end{array}$} \\
\hline & \multirow{3}{*}{\multicolumn{2}{|c|}{$\begin{array}{l}\text { Legend } \\
\text { o colonised patients } \\
\text { o nosocomial patients } \\
\text { o uncolonised patients } \\
\text { o empty beds } \\
\text { patients in single bed } \\
\text { rooms }\end{array}$}} \\
\hline 000000 & & \\
\hline 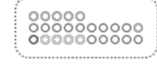 & & \\
\hline
\end{tabular}

Figure 14.3 The hospital and ward management view of our model of MRSA endemicity and its control in hospitals. 
model. System dynamics was used to model patient flow. Patient bed allocation to single-bed rooms and shared rooms was modelled using agent-based modelling methods.

3. The hospital model encapsulates four replicated ward models that have been calibrated to reflect surgical, intensive care, medical and rehabilitation ward specialties. This model simulates patient transfers between ward specialties. The average MRSA prevalence and incidence rates in each ward speciality and in the hospital overall are collected to describe the current state of MRSA in the hospital.

The interactive visual interface was designed to assist ward- and hospital-level management and to improve understanding of MRSA transmission and its control. The hospital management view provides a direct comparison of the average daily MRSA prevalence and incidence rate for each ward speciality, and shows changes in these levels over time (see Figure 14.3a). The ward management view (see Figure 14.3b) also displays how beds in the ward (circles) are allocated to MRSA colonised and susceptible patients. It highlights when a patient acquires MRSA during their stay and whether there are empty beds. Beds that are in shared rooms are differentiated from beds in single-bed rooms (within the box). These views visually describe the state of MRSA in each ward speciality and for the hospital as a whole.

Decision makers and learners can simulate alternative scenarios and generate different MRSA outcomes by modifying several infection control, ward and disease variables (see Figure 14.4). These variables can be modified before the simulation

\section{haisim}

Intensive care units

WARD SETTINGS

INFECTION CONTROL

Hand hygiene

staff compliance

efficacy for pathogen removal

Patient screening

$\%$ of patients screened on admission

$\%$ of patients screened using rapid PCR

delay for culture test results (days)

Patient isolation

$\%$ of all beds in single bed rooms efficacy in reducing probability of MRSA

transmission upon contact

Staff cohorting

(note for 1:1 hcw-to-patient ratio only)

$\%$ of staff cohorted

view all wards

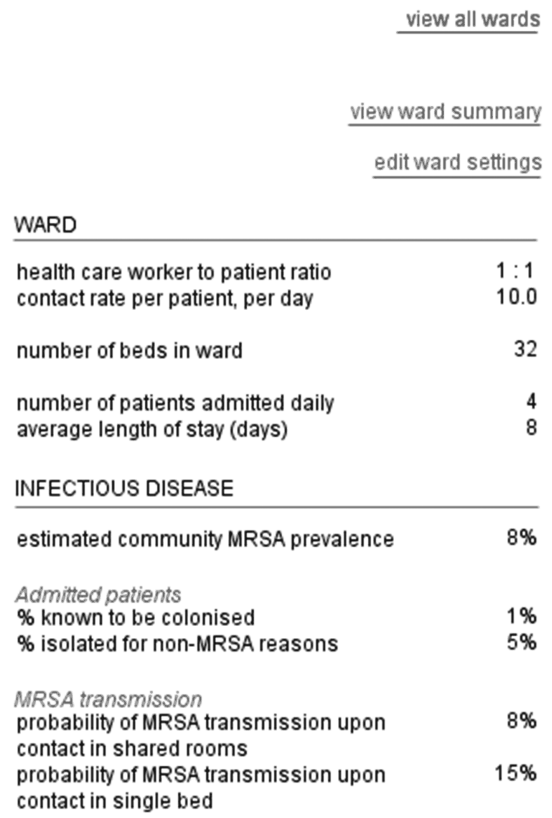

Figure 14.4 The context and decision parameters for a ward in our model of MRSA endemicity and its control in hospitals. 
begins or while the simulation is running. The effect of changing these variables on the prevalence and incidence rate of MRSA for different ward specialties can be explored. Computer simulations provide immediate feedback to the learner, encourage critical reflection on decisions, and can promote consensus for practice.

\subsubsection{Conclusion}

We demonstrate that the multilevel systems approach of Chapter 13 can be applied to develop a hybrid, multiscale simulation model of MRSA endemicity in hospitals and its control. Using this approach, key mechanisms of MRSA endemicity in hospitals and their controlling contextual factors were identified across different levels of the health system and discussed as potential areas for infection control strategies to target. The multilevel conceptual model indicates that the infection control policies studied address the incidence of MRSA in hospitals directly. There is opportunity to address the prevalence of MRSA directly as a complementary strategy. The multiscale simulation model enables ward- and hospital-level infection control strategies to be employed and explored for different ward settings. Immediate feedback in response to decisions promotes learning and multilevel perspectives encourage a collaborative response to address MRSA endemicity in hospitals.

\subsection{Chlamydia composite model}

\subsubsection{Introduction}

Sexually transmitted infections (STIs) are a continuing problem in the UK with many genito-urinary medicine (GUM) clinics being overstretched due to increases in many STIs, in particular chlamydia. A discrete-event simulation (DES) model of a Sexual Health Clinic was produced, to explore different clinic configurations to improve efficiency and increase the number of patients treated. A system dynamics (SD) model was produced to capture chlamydia transmission and screening taking into account age-specific transmission rates and different risk groups. The SD model contained a variable representing those treated as a result of being screened. This was replaced with the DES model of the Sexual Health Clinic. This incorporated a degree of detail (randomness) in the SD model of chlamydia and feedback more explicitly in the DES model of the Sexual Health Clinic. The work was conducted at St Mary's Hospital in Portsmouth from 2006 to 2010.

\subsubsection{Chlamydia}

Chlamydia is the most common bacterial STI in the world. It can be transmitted via sexual contact, and can be passed from a mother to baby during vaginal childbirth (NCSP, 2011). Chlamydia is in many cases asymptomatic, presenting with no symptoms in $75 \%$ women and $50 \%$ men (FPA, 2006). Chlamydia can result in serious consequences (sequelae), including: pelvic inflammatory disease (PID) for women, which can result in infertility and ectopic pregnancy which is potentially fatal; and, for men, possibly infertility and inflammation of the testicles. It is estimated 

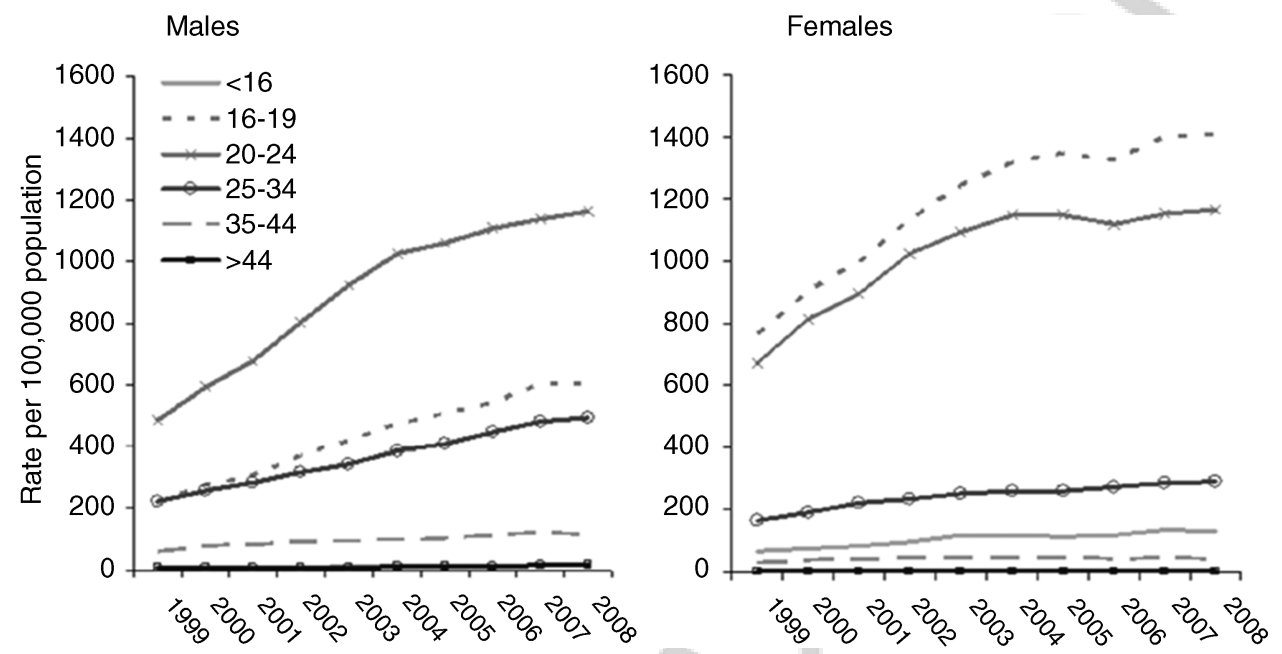

Figure 14.5 Rates of diagnoses of uncomplicated genital chlamydial infection by sex and age group, GUM departments, UK: 1999-2008 (HPA, 2007).

that the costs that result from the development of these sequelae cost the National Health Service (NHS) at least $£ 100$ million per year.

Figure 14.5 illustrates that the rates of diagnoses of uncomplicated genital chlamydial infection have been increasing in the UK since 1999. As can be seen, the rate of chlamydial diagnoses has increased disproportionately among the younger age groups (HPA, 2007).

This makes early identification and prevention vital in maintaining viable reproductive capabilities. Prevention of PID and the protection of fertility were key factors in the development of the government's sexual health strategy (Department of Health, 2001). Sexual health was also a key component of many white papers: for example, 'Choosing health: our health, our care, our say' (Department of Health, 2006) and 'Choosing health: making healthy choices easier' (Department of Health, 2004). These papers recommended the use of screening for chlamydia with a particular focus on the young (those under 25) who carried a burden of the infection.

\subsubsection{DES model of a GUM department}

DES was chosen as the preferred tool for modelling the walk-in clinic at St Mary's Hospital. DES has been used successfully in many other models of outpatient departments (Jun, Jacobson and Swisher, 1999; Fone et al., 2003).

The model allowed the department to assess different configurations that it was interested in, for example changing the number of staff and rooms, time to complete tasks, opening hours, and so on. The model was produced in collaboration with the staff within the department. The structure of the system, key performance indicators and the data for the model were agreed. Patients missed and therefore potentially missed conditions were chosen as the key performance indicators. 
Data was manually collected, screening data was analysed, and data was also sought from the Health Protection Agency (HPA), National Chlamydia Screening Programme (NCSP) and the Department of Health (DH).

\subsubsection{SD model of chlamydia}

SD was chosen as the preferred tool for modelling the transmission of chlamydia in Portsmouth and the surrounding area due to the size of the population and the long time horizon the model runs over (Townshend and Turner, 2000; Evenden et al., 2006).

The model's primary purpose is to evaluate screening programmes including: screening younger age groups with contact tracing; screening all women with contact tracing; and screening both genders with contact tracing. This model captures behavioural change in terms of changes as the people in the model age, but there is also the probability of changing behaviour following treatment.

Data for the SD model was obtained from multiple sources: the population (Office of National Statistics); probability of infection (Turner et al., 2006); contact rate (Mercer et al., 2009; Townshend and Turner, 2000); mean time to recovery (Evenden et al., 2006; NCSP, 2011); and from the core group (Zenilman et al., 1999).

\subsubsection{Why combine the models}

A composite model was constructed for the following reasons:

1. Health care models tend to focus on a specific area and do not take into account the wider system in terms of both upstream and downstream effects. The wider system can be incorporated in the composite model.

2. Models of different levels can interact and have been constructed in other industries specifically to do this. Why can the principle not be applied to health care?

Figure 14.6 gives a simple overview of the approach chosen for this work.

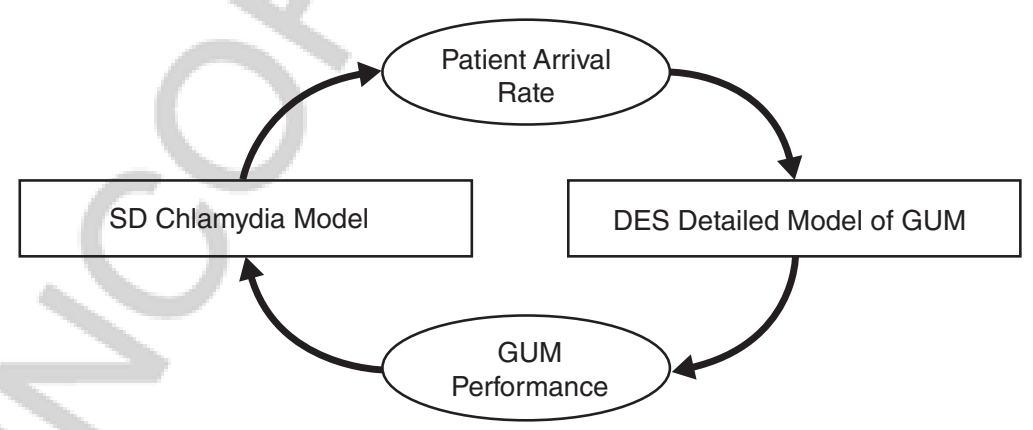

Figure 14.6 Overview of composite approach. 


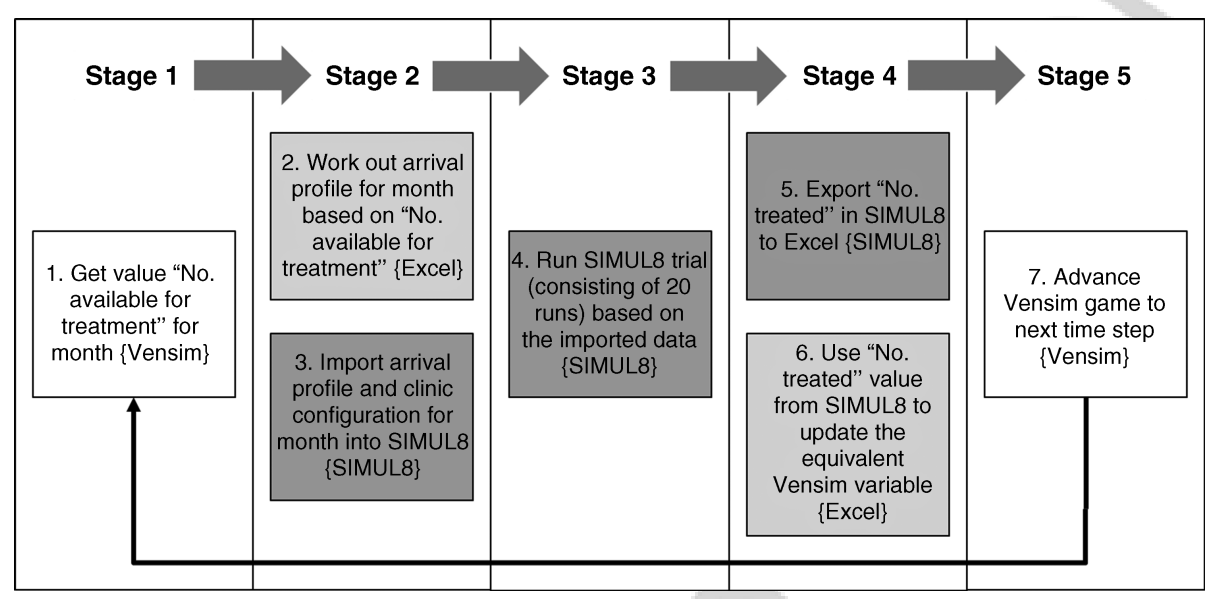

Figure 14.7 Stages involved in linking the SD and DES models.

\subsubsection{How the models were combined}

The models were constructed in commercially available packages SIMUL8 (DES) and Vensim (SD). It is possible to create interfaces and to communicate with both of these packages with VBA, through Microsoft Excel. How the models communicate and interact with one another is shown in Figure 14.7.

\subsubsection{Experiments with the composite model}

When the models are combined this dramatically increases the size of the scenario landscape.

The composite experiments are shown in Table 14.1. The staff rows in the table relate to full time equivalents (FTEs). The example row means that the department would have one member of staff working each day the department is open (without taking any breaks). The waiting area relates to the number of physical resources, waiting area chairs (capacity), etc.

To demonstrate the results from the composite model, two graphs have been chosen. Figure 14.8 represents the cumulative number of people infected over time

Table 14.1 DES parameters for the three experiments.

\begin{tabular}{|c|c|c|c|c|c|c|c|c|c|c|}
\hline & \multicolumn{5}{|c|}{ Staff } & \multicolumn{5}{|c|}{ Waiting areas } \\
\hline & Reception & Female & Male & Blood & $\mathrm{Lab}$ & Reception & Standing & Female & Male & Shared \\
\hline Example & 5.00 & 5.00 & 5.00 & 5.00 & 5.00 & & & & & \\
\hline Base & 14.91 & 10.50 & 7.73 & 4.75 & 4.75 & 4.00 & 10.00 & 10.00 & 10.00 & No \\
\hline Optimal & 28.48 & 14.24 & 14.24 & 4.75 & 4.75 & 4.00 & 10.00 & 10.00 & 10.00 & Yes \\
\hline Max & 47.46 & 47.46 & 47.46 & 4.75 & 4.75 & 4.00 & 10.00 & 10.00 & 10.00 & Yes \\
\hline
\end{tabular}




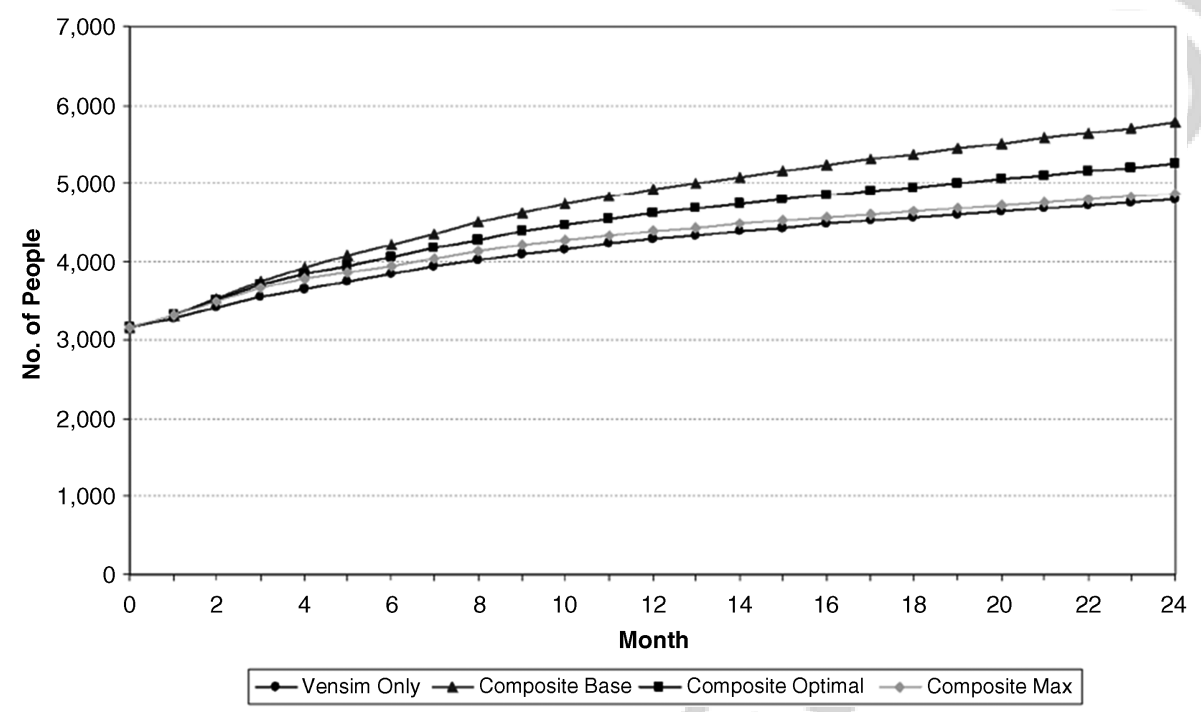

Figure 14.8 Cumulative number of people infected by month comparing composite and SD only models.

(results from the SD model), while Figure 14.9 represents the number of people not seen at the GUM department over time (results from the DES).

Figure 14.8 illustrates that by combining the DES and SD models there is an impact on the number of people who are infected over time. This is due to the

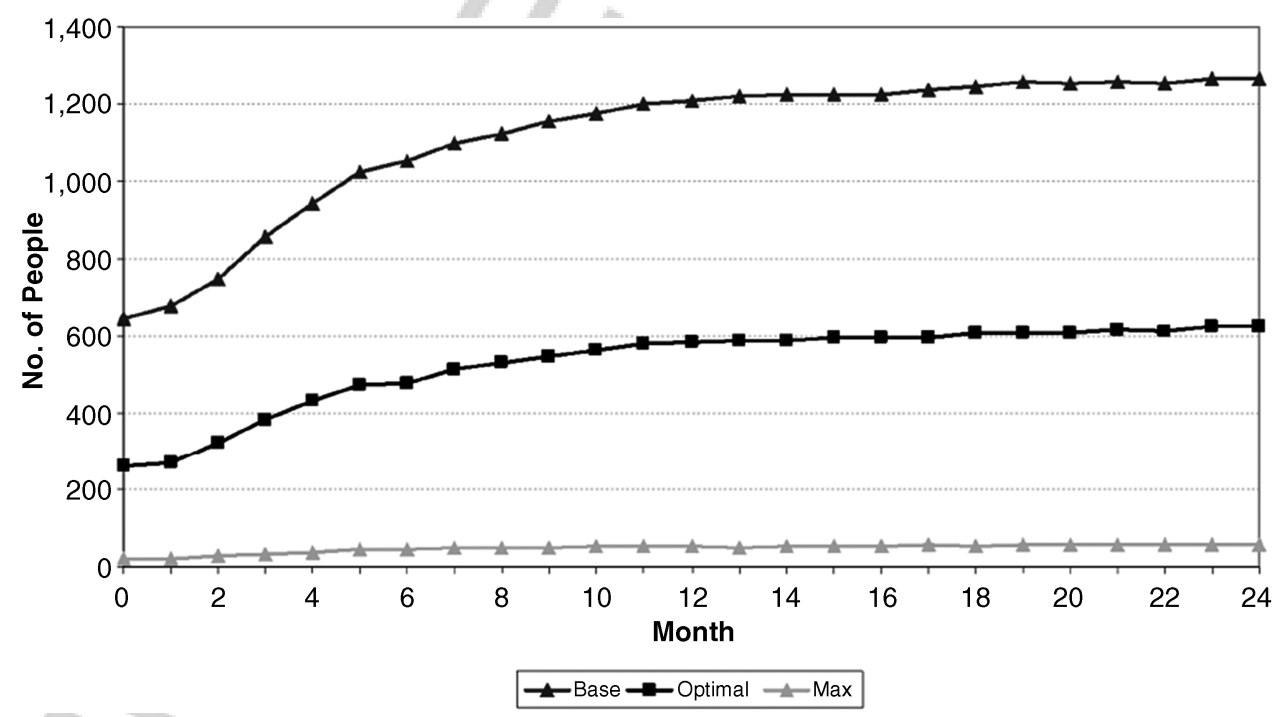

Figure 14.9 DES composite results, number of patients not seen by GUM. 
configuration of the GUM department. If the GUM department is limited due to its resources then more people become infected irrespective of the level of screening, as people are unable to be treated. This is illustrated further in Figure 14.9 which clearly demonstrates that the base configuration of the GUM department results in significantly more people not being seen and subsequently potentially returning to the wider community to further spread the infection.

\subsubsection{Conclusions}

The key outcomes derived from the composite model are as follows:

- The composite model illustrates that decisions made at the GUM level have consequences in the population that the GUM department serves and these can be quantified based on the assumptions made.

- By making savings at the GUM department configurations can still be devised to make effective use of the resources available, but this has an effect on the prevalence of chlamydia in the population, which can now be accounted for.

- By combining the models in this way feedback normally associated with SD modelling is incorporated in the DES model.

- The SD model benefits by incorporating the detail usually associated with DES modelling.

Many have spoken about and suggested the use of hybrid simulation in various industries. This is one of a few working models in health care which does not simply mention the possibility of combining the models, but actually works in practice. The communication between the two modelling approaches is fully automated, but to make interaction easier the new model has been semi-automated to enable the DES model to be updated in between the time steps of the SD model.

There are potentially many ways in which SD and DES can be combined. The amount of and how frequently the information is exchanged are dependent upon the purpose of the combined models produced and what outcomes are required. It is important to note that the composite model produced here was not created as a replacement for existing techniques. The technique was devised to complement existing modelling approaches and to be used when a problem does not lend itself solely to one particular modelling approach.

Through the development of the composite model presented in this chapter, potential avenues for further work include:

- exploration of the timings and mechanisms through which the DES and SD models communicate and exchange information;

- evaluation of the combination of other forms of modelling including: agentbased simulation (ABS), SD, DES and other forms of model;

- further evaluation of the composite modelling approach in relation to health care and other settings; and 
- comparing the development of combined models from scratch compared with the integration of software packages.

\subsection{A hybrid model for social care services operations}

\subsubsection{Introduction}

The ageing population presents many significant challenges for social care services at both a national and local level, one of which is to meet the demand for long-term care. It has been predicted that the population aged over 65 in the UK will continue to grow for some time. Social care consists of help with the activities of daily living provided to anyone with a chronic condition, that is an ongoing condition or disease that limits a person's ability to carry out everyday tasks. The concern for the government at both the national and local level is whether there will be enough resources in place to handle the expected strain on the system in the future. A study was undertaken with a local authority in the south of England, namely Hampshire County Council (HCC), to assist them with their planning. People aged over 65 are a major client group for the Council and it was this client group that was the concern of the research.

HCC have begun addressing the issue through a number of initiatives, one of which is through a modernisation programme. Part of this has been the establishment of a contact centre called Hantsdirect which will help improve efficiency so that HCC can take advantage of economies of scale. The contact centre plays an important role in the provision of social care. The service has an important role as an information provider to those in need of care (not only for publicly founded services) but acts as one of the main gatekeepers to social care. It provides an initial assessment to determine if a person is eligible for care. The design, staffing and planning of the contact centre is an important concern of the Council.

Two models were built as part of the project. First, a cell-based population model was built to forecast the demand for long-term care in Hampshire from people aged 65 and over for the period 2009 to 2026 . The model uses the same logic as an SD model. DES was used to model the contact centre. The model was designed with data from HCC as well as data collected by the researcher.

The two models were combined as a hybrid model to explore the short- and longterm performance of the contact centre in the light of demographic change. The benefits and insights - of which there were many - of combining the two models were also explored in this study.

\subsubsection{Population model}

The cell-based population modelled the home and community system only. Projections for long-term care were made for the period 2009 to 2026 for people aged over 65 and living in the household population of Hampshire. It is important that HCC have a useful set of data to help plan services. This will potentially allow appropriate 
funding and resources to be allocated to meet the increases in demand. The results of this model can potentially aid the decision-making process.

The model was designed at a local level and populated with actual data from a wide range of sources including population data from the Department for Communities and Local Government (2012) and the National Statistics Subnational Population Projections Unit (2012). An important part of the model was the creation of a disability index using data from the 2001 General Household Survey (Economic and Social Data Service, 2001). This was supplemented with data from HCC.

The cell-based model uses the same logic as an SD model in terms of moving a population through different compartments. Each compartment represents an age group which is further broken down by level of disability and main service receipt.

The disability groups explored were low, moderate, substantial, critical, and the service providers modelled were formal care (HCC), informal care, no care, private care.

Due to the lack of relevant data, it was decided not to include feedback effects but to treat the model as a population projection model. This is not a weakness of the model, as the fundamental objective of projecting the demand for long-term care is still achieved. The inclusion of feedback would have relied on assumptions based solely on expert judgement. This would have been a potential weakness of the model and would have added no additional value.

\subsubsection{Model construction}

The model was constructed as a spreadsheet model as the features that were needed could be constructed within Microsoft Excel (2010) and hence the model could be easily shared with HCC. The model can be described by the two equations below. The population in the model for the end of each calendar year was calculated using equation (14.1). With

$h_{k g t}:$ the household population of age group $k(65-69,70-74,75-79,80-84,85+)$, gender $g$ (male, female) at calendar year $t$,

$u_{k g t}$ : the numbers of age group $k$, gender $g$, who have reached the highest year of the age group at year $t$,

$n_{k g t}^{1}$ : the net population change for age group $k$, gender $g$, through migration and death, for year $t$, which was derived from $n_{\text {agt }}$,

$d_{k g e}$ : the disability rate for disability category $e$ (low, moderate, substantial, critical), age group $k$, gender $g$, and

$s_{\text {kgef: }}$ the service rate for service category $f$ (formal care, informal care, no care, private care), disability category $e$, age group $k$, gender $g$,

then the population of age group $k$, gender $g$ at calendar year $t$ is given by

$$
h_{k g t}=h_{k g t-1}+u_{k-1 g t-1}-u_{k g t-1}+n_{k g t}^{1}
$$

The household population $\left(h_{k g t}\right)$ at the end of the calendar year for any age group is calculated by taking the household population for the same age group from the previous 
year $\left(\mathrm{h}_{k g t-1}\right)$ and accounting for population changes. The first population change is the number ageing from the younger age group $\left(u_{k-1 g t-1}\right)$. For example, for the age group $70-74$ in 2010, $u_{k-1 g t-1}$ is the number of people aged 69 in 2009 . The second population change is to account for the number of people ageing to the next age group $\left(u_{k g t-1}\right)$. For example, for the age group 70-74 in 2010, the number of people aged 74 from 2009 are removed from their current age group and are now added to the age group 75-79 in 2010. The final population change to account for is the net population change for the age group $\left(n_{k g t}^{1}\right)$ accounting for all the ages that are represented in that age group. For example, the net population change for the age group 65-69 accounts for migration and mortality changes for the ages $65,66,67,68$ and 69 .

Using the results of equation (14.1), the following expansions are applied in the cellbased model. First, the disability rates for each age group and gender are multiplied by the household population in the equivalent age group and gender. The population in disability category $e$, age group $k$, gender $g$ at time $t$ is given by $d_{k g e} h_{k g t .}$ Second, the proportions of service receipt for each disability category are multiplied by the number of disabled people in each age group by gender. Thus, the population in service category $f$, disability category $e$, age group $k$, gender $g$ at time $t, p_{k g e f t}$, is given by

$$
p_{\text {kgeft }}=s_{k g e f} d_{k g e} h_{k g t}
$$

This allowed the number of people with a disability to be predicted by age group, gender and the likely source of help.

\subsubsection{Contact centre model}

The contact centre was modelled using DES, which is a proven and successful approach to modelling call centres in order to test various changes and observe the impact upon performance. It allows for all the key features of Hantsdirect to be modelled. The software used was SIMUL8 (SIMUL8, 2012).

Hantsdirect has many complex features, which suggests that DES would be an appropriate approach. These features include different types of staff, time-dependent arrival times, different types of call which require different call handling times, complex routing rules, various forms of contact (telephone, e-mail, fax), outbound and abandoned calls. The purpose of modelling HCC's call centre was to be able to produce performance outputs under various demand levels. Many different performance measures can be produced from a simulation model. However, measures of particular interest in this study are:

- Distribution of time in the system (total processing time)

- Staff utilisation rates

- Number of abandoned calls

- Percentage of calls answered

- Percentage of calls answered within 20 seconds. 
The simulation model was used to test a variety of different staffing scenarios but, most importantly, it was used as part of the hybrid framework.

\subsubsection{Hybrid model}

The combination of the two models allowed the performance of the contact centre to be explored over a 10-year period (2010-2020). In addition to standard call centre statistics, the cell-based model was used to predict the increase in demand in terms of the number of calls received due to demographic change. Based on this demand, the monthly performance of the contact centre was explored through the simulation model. This performance (in terms of abandoned calls) was then fed back into subsequent runs of the DES model, by assuming that a fraction of abandoned calls would be followed by increased demand in future months.

For the purposes of the hybrid model, the cell-based model was extended to include the rest of the population, as the contact centre receives calls from all age groups. This was only in terms of population projections and not a breakdown by various levels of disability. An additional extension included accounting for people aged 65 and over and who are not members of the household population; that is, are living in an institution. Calls can be received from people in institutional care.

This hybrid framework is useful from both a theoretical and a practical perspective. The following questions are addressed by the hybrid framework:

1. How could a detailed tactical model for the contact centre benefit from the additional use of a long-term dynamic demographic model for population change?

2. What benefits and insights would result from a combined approach based on these two different models?

Figure 14.10 illustrates the hybrid framework. The cell-based model generates the number of initial contacts for the contact centre. These initial contacts are fed into the

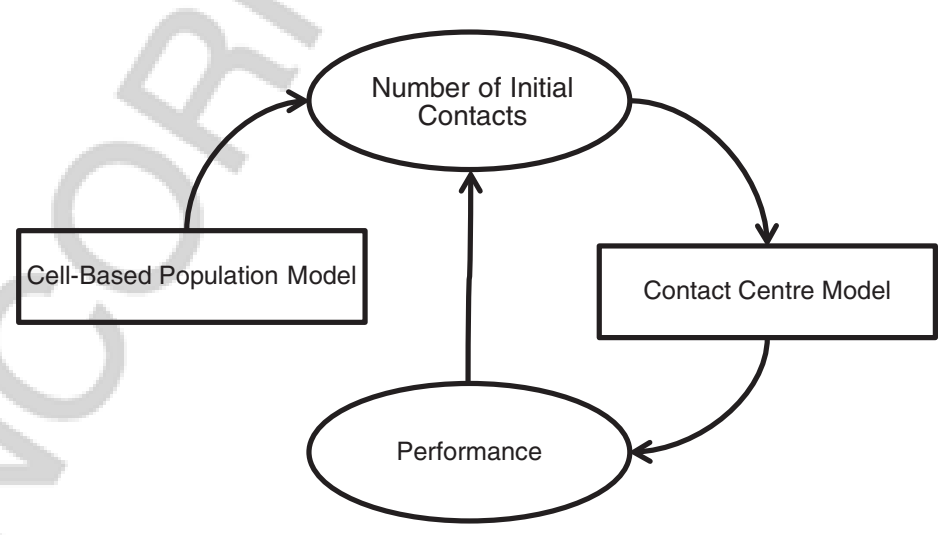

Figure 14.10 Hybrid model. 
contact centre model to complete the top half of the loop shown in Figure 14.10. Each month a number of key performance indicators (KPIs) are reported from the DES contact centre model.

One assumption that potentially could be made is that a proportion of callers who abandoned their calls would subsequently experience deterioration in their condition. This proportion is unknown but it has important implications for both the contact centre and the welfare of the people concerned. The hybrid framework accounts for the people whose situation has deteriorated and who call back later requesting help, by including them in the new initial number of client profiles. This completes the bottom half of the loop illustrated in Figure 14.10. These people are added in a month later. However, as a consequence of the delay in getting the support they need, the model assumes that disability level for people aged 65 and over will be altered. People with a slight disability will have their disability status altered to moderate, and people with a moderate disability will have their disability status altered to severe.

The two models have been combined manually using a spreadsheet as an interface to transfer data and transform data between the two models. Four main experiments have been run as part of the research.

- Experiment 1: the model is run with no inclusion of people who abandoned their calls previously. The aim was to provide a baseline against which a number of scenarios could be compared.

Since the true proportion of people who abandon their calls and then have their situation deteriorate is unknown, the following experiments were carried out:

- Experiment 2: 2\% of callers have their situation deteriorate and call back after one month.

- Experiment 3: 5\% of callers have their situation deteriorate and call back after one month.

- Experiment 4: $10 \%$ of callers have their situation deteriorate and call back after one month.

Experiments 2, 3 and 4 allow a realistic range of possibilities to be explored and provide a useful set of results. These experiments were carried out for the years 2010, 2015 and 2020. Five-year intervals were chosen to allow for significant changes in the population to occur. There was no added value for running the experiment for each year of the 10-year period.

At the time of the study, Hantsdirect aspired to meet the following two targets: that $95 \%$ of their calls are answered and $80 \%$ of their calls are answered within 20 seconds. If in any of the experiments these targets were not met, an additional two experiments were carried out. There were two types of resources working at Hantsdirect: agents and advisors. Advisors had additional skills that agents did not have, including the ability to carry out an initial assessment of care for an elderly person potentially in need of state support. The first intervention is to increase the number of agents to the point where the KPIs are achieved and the second is to increase the number of 
advisors to the point where the KPIs are once again achieved. This gives a total of nine experiments (Experiments 2, 3 and 4, each for the years 2010, 2015 and 2020). This number will increase if any staffing interventions are required.

The hybrid process involves a monthly interaction between the cell-based model and the simulation model. The call profile for the first month is used as an input for the simulation model. The call centre simulation is run with the new call arrival configuration. The simulation is run for one month and the outputs are reported after 40 trials. The simulation is a terminating simulation.

For Experiment 1, for all three of the experimental years, the same process is carried out for each of the remaining 12 months. The call profiles vary each month due to changes in the number of people in the Hampshire population.

Experiments 2, 3 and 4 allow for the inclusion of abandoned callers redialling the service. The call profile for the first month is calculated in the same way as Experiment 1. After the first month, the process changes for the remaining months. The call profiles have been altered to include the impact of abandoned callers.

For example, in month 2 of Experiments 2, 3 and 4 the call profiles include a certain percentage of additional calls. For example, in Experiment 2, 2\% of callers who abandoned their call in month 1 call back, for Experiment 3 it is $5 \%$ and for Experiment 4 it is $10 \%$. The process is replicated for the remaining 10 months. This is the same for all three experimental years. Therefore, each month the call profile varies due to changes in the population and as a result of including abandoned call feedback. Table 14.2 illustrates the results of the hybrid framework and where staffing interventions were required.

When the contact centre conditions are kept the same, the model predicts that the number of staff needs to be increased in both 2015 and 2020. Surprisingly enough, there is not predicted to be much difference between the impact of both agent and advisor interventions upon performance.

Feedback does have a slight impact upon the system for all three years and the impact increases as the number of call arrivals increases. The true impact upon performance over the test period is likely to be due to changes in the population of Hampshire.

The hybrid framework has shown to be a useful toolkit in examining the impact of increasing demand upon the performance of the contact centre. It should be noted that the results for 2015 and 2020 are highly theoretical. Much can change over the next 10 years. There are many unknown future parameters. This in no way should undervalue the importance of the experiments. It is a means of validating the decisions of the Hantsdirect manager's current staffing policies. It also highlights the importance of modelling other issues such as the impact of feedback from abandoned calls.

\subsubsection{Conclusions and lessons learnt}

The hybrid framework has had a practical impact for HCC. This research not only looks at the changing demographics of Hampshire, but also models the impact it has had on the contact centre. It allows for a more sophisticated demand profile to be created instead of simply inflating call arrival rates by allowing for the inclusion of 


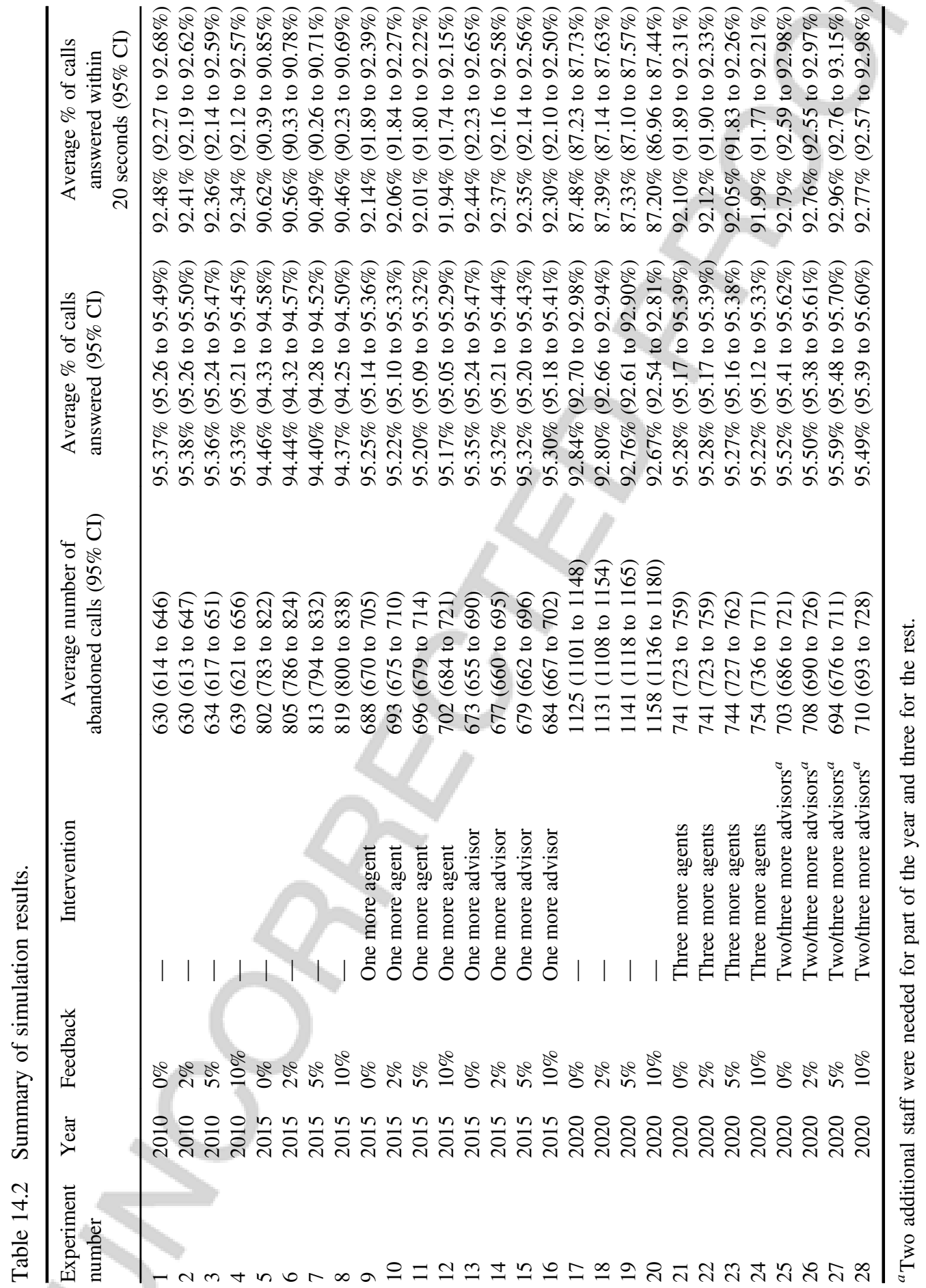


feedback created from abandoned calls from the previous month. Many existing simulation models of contact centres only look at short-term issues. Such models typically do not account for factors in the external environment. The combination of the two models permitted the exploration of performance issues as a result of longterm demographic change which is one of the main advantages of the framework.

Cell-based models (SD) and DES models alone have been proven to work successfully across many application areas, but this research shows that by having a combined framework a more robust modelling technique can be formed. The new framework has all the strengths of the original methodologies and also allows the researchers to address new questions that could not have been answered with one model alone. In this research, a DES model study of the contact centre would have only looked at the performance of the contact centre today and would not have studied the impact of changing population demographics and increased numbers of disabled people in the Hampshire population.

The two standalone models can potentially be useful for HCC. The Adult Services Department has taken ownership of the cell-based model and is using it as an additional piece of evidence in the planning of future services. The data provided the Council with a set of information they did not have before and the results of the model have the potential to improve decisions in both the short and long term.

The cell-based model was an ideal choice for modelling the Hampshire population to project future levels of disability and service receipt. The model is able to produce results almost instantaneously at the population level. This model can easily be used by HCC, as it was built in Excel, software which HCC already own and with which HCC staff are comfortable and familiar. This allows the Council to run their own experiments and also increases user acceptance of the model. It would not have been useful to provide $\mathrm{HCC}$ with the hybrid model: in addition to requiring the purchase of the SIMUL8 software and training in its use, the hybrid model requires considerable technical expertise to run.

DES models are ideal for modelling queuing systems (such as call centres) in order to understand performance under varying conditions. The cell-based model would not have been able to model the contact centre in sufficient detail and capture the stochastic aspects of the real-world system. Since the DES model was developed as a research tool and was not intended to be used by the Council, there was no need to develop a user interface or train HCC staff on how to use the SIMUL8 software. The model was used to explore various staffing scenarios that were not part of the hybrid framework. Hantsdirect requested these additional experiments over and above the modelling that was carried out to address the research questions in this section. Since the DES model was separate from the hybrid framework, these experiments were rapidly and easily carried out. The results can be potentially useful for resource planning.

As the two models have been shown to be useful on their own, it was not considered important to explore the possibility of building the model in one environment. This would have been a time-consuming programming task, and only of value if HCC had requested a user-friendly software tool which did this. Moreover, by validating the models separately, we did not need to worry about validating the combined model. The two individual models were validated using standard validation 
and verification approaches. Much time would be needed to ensure that the single environment model was fully validated before the results could be relied on.

The hybrid framework potentially helps decision making in a number of issues which would not have been possible through the use of either one of these models on their own, through its ability to address both strategic and operational issues, and provides a more robust approach.

\section{References}

CDC (Centers for Disease Control and Prevention) (2011) Healthcare-associated infections (HAI), http://www.cdc.gov/hai/index.html (accessed 12 November 2012).

Department for Communities and Local Government (2012) http://www.communities.gov.uk/ corporate/ (accessed 27 May 2012).

Department of Health (2001) National strategy for sexual health and HIV, www.dh.gov.uk. Department of Health (2004) Choosing health: making healthy choices easier, www.dh.gov.uk. Department of Health (2006) Choosing health: our health, our care, our say, www.dh.gov.uk. Economic and Social Data Service (2001) www.esds.ac.uk/ (accessed 27 May 2012).

Evenden, D., Harper, P.R., Brailsford, S.C. and Harindra, V. (2006) Improving the costeffectiveness of Chlamydia screening with targeted screening strategies. Journal of the Operational Research Society, 57(12), 1400-1412.

Ferlie, E.B. and Shortell, S.M. (2001) Improving the quality of health care in the United Kingdom and the United States: a framework for change. Milbank Quarterly, 79(2), 281-315.

Fone, D., Hollinghurst, S., Temple, M. et al. (2003) Systematic review of the use and value of computer simulation modelling in population health and health care delivery. Journal of Public Health, 25(4), 325-335.

Forrester, J.W. (1961) Industrial Dynamics, MIT Press, Cambridge, MA.

FPA (2006) Chlamydia: looking after your sexual health, Pamphlet.

Glass, T.A. and McAtee, M.J. (2006) Behavioral science at the crossroads in public health: extending horizons, envisioning the future. Social Science Medicine, 62(7), 1650-1671.

Gould, I.M. (2005) The clinical significance of methicillin-resistant Staphylococcus aureus. Journal of Hospital Infection, 61(4), 277-282.

Grundmann, H., Aires-de-Sousa, M., Boyce, J. et al. (2006) Emergence and resurgence of methicillin-resistant Staphylococcus aureus as a public-health threat. The Lancet, 368(9538), 874-885.

Hamer, W.H. (1906) The Milroy lectures on: Epidemic disease in England - the evidence of variability and of persistency of type. The Lancet, 167(4307), 733-739.

HPA (Health Protection Agency) (2007) All new STI episodes seen at GUM clinics in the UK 1998-2007, www.hpa.org.uk/STIannualdatatables (accessed 20 September 2008).

Jun, J.B., Jacobson, S.H. and Swisher, J.R. (1999) Application of discrete-event simulation in health care clinics: a survey. Journal of the Operational Research Society, 50(2), 109-123.

Kermack, W.O. and McKendrick, A.G. (1927) A contribution to the mathematical theory of epidemics. Proceedings of the Royal Society, 115, 700-721.

Köck, R., Becker, K., Cookson, B. et al. (2010) Methicillin-resistant Staphylococcus aureus (MRSA): burden of disease and control challenges in Europe. Euro Surveillance, 15(41), 
www.eurosurveillance.org/ViewArticle.aspx?ArticleId=19688 (accessed 12 November 2012).

Mercer, C.H., Copas, A.J., Sonnenberg, P. et al. (2009) Who has sex with who? Characteristics of heterosexual partnerships reported in a national probability survey and implications for STI risk. International Journal of Epidemiology, 38, 206-214.

National Statistics Subnational Population Projections Unit (2012) http://www.ons.gov.uk/ ons/index.html (accessed 27 May 2012).

NCSP (2011) National Chlamydia Screening Programme, http://www.chlamydiascreening .nhs.uk/ps/ (accessed 5 January 2011).

Object Management Group (OMG) (2012) ISO/IEC 19505-2: Information technology - Object Management Group Unified Modeling Language (OMG UML) - Part 2: Superstructure, http://www.omg.org/spec/UML/ISO/19505-1/PDF (accessed 12 November 2012).

Pawson, R. and Tilley, N. (1997) Realistic Evaluation, Sage, London.

SIMUL8 (2012) www.simul8.com/ (accessed 27 May 2012).

Sterman, J.D. (2000) Business Dynamics: Systems Thinking and Modeling for a Complex World, Irwin/McGraw-Hill, New York.

Stommel, H. (1963) Varieties of oceanographic experience. Science, 139(3555), 572-576.

Townshend, J.R.P. and Turner, H.S. (2000) Analysing the effectiveness of Chlamydia screening. Journal of the Operational Research Society, 51(7), 812-824.

Turner, K.M.E., Adams, E.J., Gay, N. et al. (2006) Developing a realistic sexual network model of Chlamydia transmission in Britain. Theoretical Biology and Medical Modelling, 3(3), 3.

WHO (World Health Organization) (2011) Report on the burden of endemic health careassociated infection worldwide, http://www.who.int/gpsc/country_work/burden_hcai/en/ index.html (accessed 12 November 2012).

Zenilman, J., Ellish, N., Fresa, A. and Glass, G. (1999) The geography of sexual partnerships in Baltimore: applications of core theory dynamics using a geographic information system. Sexually Transmitted Diseases, 26(2), 75-81. 